\title{
Microfluidic Device for Sequential Injection and Flushing of Solutions and its Application to Immunosensing
}

\author{
Norihiro Nashida* Non-member \\ Hiroaki Suzuki* Member
}

\begin{abstract}
A microfluidic system with injecting and flushing functions was developed. In the system, hydrophilic flow channels have a dry-film photoresist layer which facilitates the introduction of solutions from four injection ports. The injection and flushing of solutions are controlled by valves operated by electrowetting. The valves consist of gold working electrodes in the flow channels or a through-hole in the glass substrate. Solutions can be sequentially introduced through the injection ports into a reaction chamber and flushed through a valve in the through-hole. Necessary immunoassay steps can be conducted on the chip, and a target antibody can be detected electrochemically.
\end{abstract}

Keywords : electrowetting, valve, hydrophilic flow channel, immunoassay

Over the last decade, research on the micro total analysis system ( $\mu$ TAS) or Lab-on-a-Chip has advanced dramatically, with the focus on point-of-care testing and on-site analysis. As development progresses, one of the ultimate goals is the integration of microfluidic transport function. The size of the microfluidic components is being decreased; therefore, the use of interfacial tension as a driving force is becoming one of the potential options for microfluidic transport. In this respect, electrowetting provides a promising methodology for the construction of an efficient microfluidic transport system ${ }^{(1)}$. In this study, we used electrowetting to realize a mechanism for introducing solutions sequentially and flushing them after use.

Immunoassay, one of the most powerful techniques in medical science, food processing, environmental monitoring, and other fields, requires the sequential introduction of solutions for incubation and rinsing. Although a group at the University of Cincinnati has reported on-chip immunoassay using integrated magnetically actuated microvalves ${ }^{(2)}$, there have been few such trials, possibly due to the difficulty in realizing microfluidic devices. The structure and operation of our fabricated device are simple, facilitating the handling of many solutions.

Our system consists of a glass substrate with flow channels, a reaction chamber, and a PDMS substrate with four injection ports (Fig. 1). First, a through-hole was sandblasted into the glass substrate to make a flushing valve. A gold layer was then deposited on the glass substrate, with a chromium intermediate layer added to form a base. Gold working electrodes for the valves were installed. Platinum and silver layers were also deposited and patterned to form a working electrode and a reference electrode for detection. The active areas of the electrodes were delineated with polyimide, and the glass substrate in the channel area was exposed. After the electrodes were formed, channel patterns were formed with a dry-film photoresist (Hitachi Chemical Company, ME-1050EA). The height of the channels is $50 \mu \mathrm{m}$, and the diameter of the chamber is $2.4 \mathrm{~mm}$. The width of the flow channels connecting the injection ports and the reaction chamber is $200 \mu \mathrm{m}$. The channel was sealed with a PDMS substrate, and

\footnotetext{
* Graduate School of Pure and Applied Sciences, University of Tsukuba, 1-1-1 Tennodai, Tsukuba, Ibaraki 305-8573
}

the necessary structures were formed using an SU-8 (MicroChem) template. Recesses $65 \mu \mathrm{m}$ deep, the walls of which were coated with PDMS, were formed in the valve areas to prevent the uncontrolled entrance of solutions into the chamber (Fig. 1). Through-holes were formed in the PDMS substrate at the ends of the flow channels to make the injection ports. A compartment with a filter paper was placed under the glass substrate. Finally, goat anti-human $\alpha$-fetoprotein (AFP) antibodies were immobilized in a plasma-polymerized film (PPF) on the platinum electrode using hexamethyldisiloxane as a monomer ${ }^{(3)}$.

The procedure for introducing and flushing a solution is illustrated in Fig. 2. When a solution is filled in an injection port, the solution moves in the hydrophilic flow channel. However, the

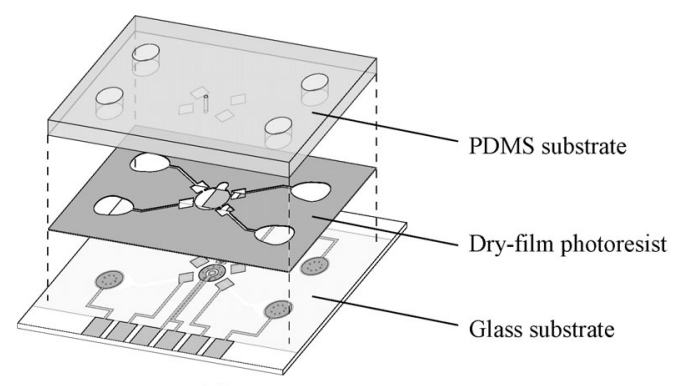

(a)

Reference electrode for transporting a solution $(\mathrm{Ag} / \mathrm{AgCI})$

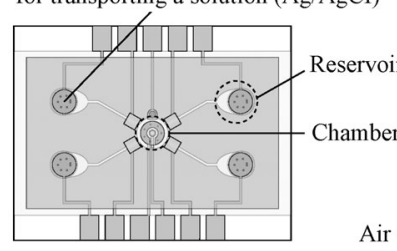

(b)

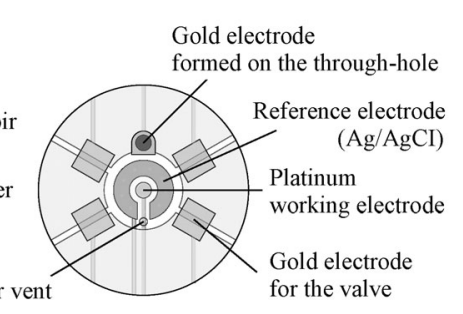

(c)
Fig. 1. Structure of the system. (a): Decomposed structure. The compartment with the filter paper is not described. (b): Top view of the completed system. (c): Magnified view of the area used for immunosensing. The dimensions of the chip are $22 \mathrm{~mm} \times 17 \mathrm{~mm}$ 


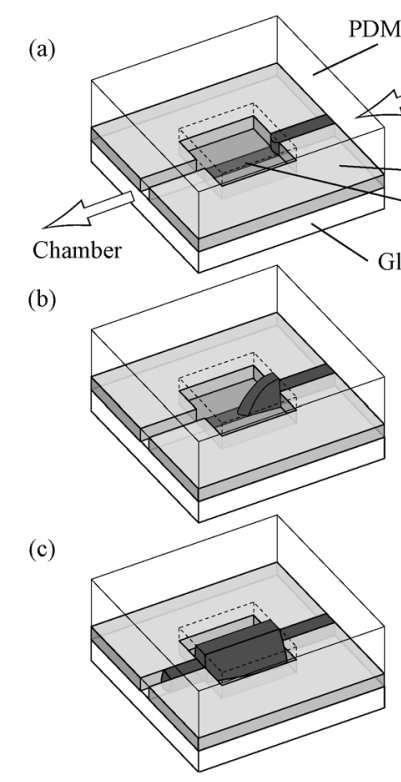

(e)

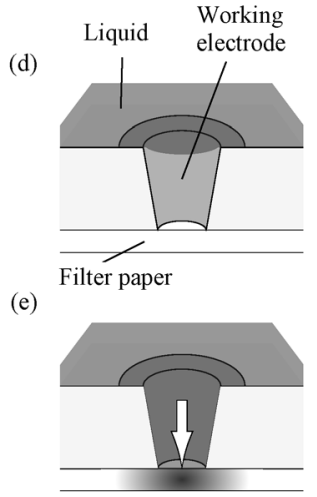

Fig. 2. Function of the valves used to inject $((a) \sim(c))$ and flush ((d) and (e)) solutions. (a), (d): Before the potential is applied. (b), (c), (e): After the potential is applied

meniscus of the solution stops in the valve area because the surface of the gold layer is initially not sufficiently hydrophilic and the volume of the area is large (Fig. 2 (a)). When $-0.9 \mathrm{~V}$ (vs. $\mathrm{Ag} / \mathrm{AgCl})$ is applied to the working electrode, the electrode becomes hydrophilic and the solution passes through the valve area and moves spontaneously into the hydrophilic area (Fig. 2 (b), (c)). After the reaction chamber is filled, the solution does not drop through the flushing valve because it is not sufficiently hydrophilic (Fig. 2 (d)). However, when $-0.9 \mathrm{~V}$ is applied to the working electrode in the flushing valve, the electrode becomes hydrophilic and the solution crosses the gold electrode area (Fig. 2 (e)). The solution is rapidly absorbed in the underlying filter paper, and the chamber is emptied.

To demonstrate the system's applicability to immunosensing, rabbit anti-goat IgG labeled with glucose oxidase (GOD) was used as an analyte. First, $1.0 \mu \mathrm{L}$ of the IgG solution, $2.0 \mu \mathrm{L}$ of a $0.1 \mathrm{M}$

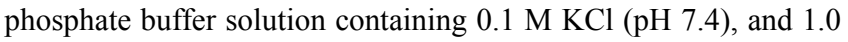
$\mu \mathrm{L}$ of a $0.1 \mathrm{M}$ phosphate buffer solution containing $0.1 \mathrm{M} \mathrm{KCl}$ and $0.3 \mathrm{M}$ glucose ( $\mathrm{pH}$ 7.4) were filled in three of the four injection ports. Next, the labeled IgG solution was introduced into the reaction chamber, and the antibodies were allowed to react with the immobilized antibodies for $10 \mathrm{~min}$. After the mixed solution was flushed, the phosphate buffer solution was introduced and flushed to remove unbound antibodies. Finally, the glucose solution was introduced into the chamber. The hydrogen peroxide produced by the enzymatic reaction of GOD was oxidized electrochemically on the platinum electrode by applying $+0.7 \mathrm{~V}$ (vs. $\mathrm{Ag} / \mathrm{AgCl}$ ), and the generated current was detected by the sensor.

With the valves in the flow channels switched on, solutions in the injection ports were sequentially introduced into the reaction chamber. The solution used in each step was subsequently flushed out of the reaction chamber by switching on the flushing valve. Since an air vent had been formed in the PDMS substrate, the filling of the solution into the reaction chamber was very smooth.

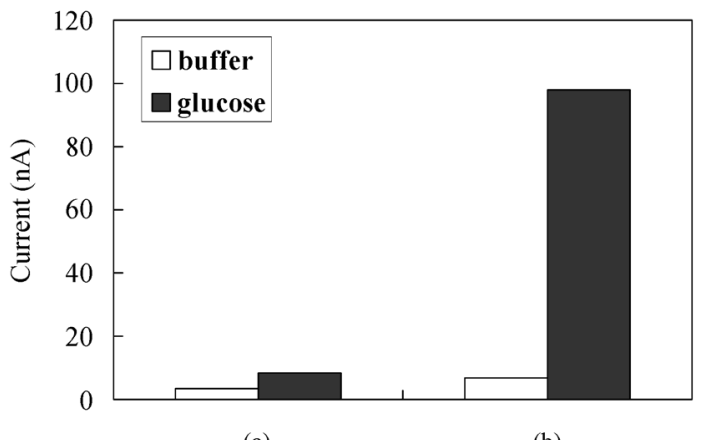

Fig. 3. Influence of nonspecific adsorption. (a): Only the PPF was deposited without the antibodies. (b): 0.2 $\mu \mathrm{L}$ of goat anti-AFP antibodies $(100 \mu \mathrm{g} / \mathrm{mL})$ was immobilized

Therefore, a stable output current was always detected by the sensor. Since the operation of the valves is based on direct electrowetting, the movement of the solution was always unidirectional. When the solution was flushed from the chamber, the solution extending from the injection port to the chamber was pulled from both sides. As a result, the liquid column was cut at the wide valve area, and the solution between the area and the chamber was flushed. The flushing valve could be used repeatedly after it was dried.

The device was successfully used to sequentially introduce solutions necessary for immunoassay. When the potential was applied to the working electrode for sensing after the labeled antibody was bound, the current stabilized within a few minutes. When the phosphate buffer solution was introduced, the current gradually decreased and settled at $7 \mathrm{nA}$. On the other hand, when the $0.3 \mathrm{M}$ glucose solution was introduced, the stabilized current increased markedly to $98 \mathrm{nA}$. In this study, no blocking agent was used. Therefore, the nonspecific adsorption of the labeled antibodies to PPF might pose a problem. As shown in Fig. 3 (a), the output current was obtained without the immobilized antibodies. In contrast to the case with immobilized antibodies (Fig. 3 (b)), the current increase was very small, suggesting that the nonspecific adsorption was negligible and the chamber was sufficiently cleansed with the phosphate buffer solution.

With this kind of microsystem, repeated use for handling physiological solutions such as whole blood would be difficult; however, the simple structure and function of our device would enable disposable use.

(Manuscript received Sep.1, 2005, revised Dec. 26, 2005)

\section{References}

(1) W. Satoh, H. Hosono, and H. Suzuki : "On-chip microfluidic transport and mixing using electrowetting and incorporation of sensing functions", Anal. Chem., Vol.77, pp.6857-6863 (2005)

(2) J. W. Choi, K. W. Oh, A. Han, N. Okulan, C. A. Wijayawardhana, C. Lannes, S. Bhansali, K. T. Schlueter, W. R. Heineman, H. B. Halsall, J. H. Nevin, A. J. Helmicki, H. T. Henderson, and C. H. Ahn : "Development and characterization of microfluidic devices and systems for magnetic bead-based biochemical detection", Biomed. Microdevices., Vol.3, pp.191-200 (2001)

(3) K. Kojima, A. Hiratsuka, H. Suzuki, K. Yano, K. Ikebukuro, and I. Karube : "Electrochemical protein chip with arrayed immunosensors with antibodies immobilized in a plasma-polymerized film", Anal. Chem., Vol.75, pp.1116-1122 (2003) 